\title{
PI-QUAL v.1: the first step towards good-quality prostate MRI
}

\author{
Maarten de Rooij ${ }^{1}$ Jelle O. Barentsz ${ }^{1}$ (1)
}

Received: 22 September 2021 / Accepted: 13 October 2021 / Published online: 29 November 2021

(c) The Author(s), under exclusive licence to European Society of Radiology 2021

\section{Key Points}

- It is mandatory to evaluate the image quality of a prostate MRI scan, and to mention this quality in the report.

- PI-QUAL $v 1$ is an essential starting tool to standardize the evaluation of the quality of prostate MR-images as objectively as possible.

- PI-QUAL will step by step develop into a reliable quality assessment tool to ensure that the first step of the MRIpathway is as accurate as possible.

Prostate MRI is recommended as a primary diagnostic test for men suspected of prostate cancer (PCa) [1]. An MRIguided diagnostic pathway is supported by Level 1 evidence in Western populations [2-4]; its high negative predictive value and sensitivity allow safe avoidance of unnecessary prostate biopsy and reduce overtreatment of indolent tumors. In addition, MRI can reliably identify clinically significant cancer suspicious regions for targeted biopsy, thereby optimizing tissue sampling.

The associated downside of the increased use of prostate MRI is variable image quality between centers and scanners [5]. Good-quality prostate MRI is the starting point and prerequisite for optimal patient management, which affects all downstream steps in the diagnostic pathway [6]. Sub-optimal image quality can lead to decreased diagnostic accuracy and increased uncertainty. Experts of the European Society of Urogenital Radiology (ESUR) and the EAU Section of Urologic Imaging (ESUI) stressed the importance of image quality assessment in a consensus document on quality requirements for image acquisition, interpretation, and training of radiologists [7]. One of the consensus-based recommendations was to assess the image quality of the MRI scan and to mention this in the report. The image quality assessment was suggested to consist of a visual analysis of the scan by the radiologists.

Giganti and colleagues addressed this problem by developing a 5-point Likert scale Prostate Imaging Quality (PIQUAL) assessment system, which ranges from very poor (PI-QUAL 1) to excellent image quality (PI-QUAL 5) [8, 9]. The assessment is based on adherence to both the technical recommendations of the Prostate Imaging Reporting and Data System (PI-RADS) and more subjective image quality criteria to score T2-weighted imaging (T2W), diffusionweighted imaging (DWI), and dynamic contrast-enhanced MRI (DCE) [10]. The authors must be commended for paving the way for standardizing prostate image quality. Development of such an assessment system with subjective criteria that are tried to be as objective as possible is not only important but also challenging. The paper by Giganti and colleagues in the current issue of European Radiology [11] shows promising reproducibility rates of their PI-QUAL v.1 assessment system with a strong weighted kappa (0.82) when clustering PI-QUAL in three quality groups (PI-QUAL 1-2 vs PI-QUAL 3 vs PI-QUAL 4-5).

When studying their results, there are several remarks that should be recognized in the next version of PI-QUAL (v.2).

This comment refers to the article available at https://doi.org/10. 1007/s00330-021-08169-1.

\footnotetext{
Jelle O. Barentsz

Jelle.barentsz@radboudumc.nl

1 Department of Medical Imaging, Radboud University Medical Center, P.O. Box 9101, Nijmegen 6500 HB, The Netherlands
} 
Firstly, the proposed PI-QUAL v.1 assessment automatically includes clinical implications into the observed image quality score. For example, a PI-QUAL score of 4 and higher implies that prostate image quality is high enough to rule in and rule out all clinically significant lesions, while this is impossible when an examination that is assessed as PIQUAL $\leq 2$. It is indeed important to make recommendations on the clinical implications of an overall assessment, but deriving this automatically from the observed PI-QUAL assessment may not be helpful in all clinical scenarios. A large suspicious lesion can be detected even in an unsatisfactory quality scan (PI-QUAL 1), while a small significant tumor can be missed on a good-quality image (PI-QUAL 4). Thus, a two-step approach seems more logical. The first step involves an assessment system that evaluates image quality as objective as possible without a derived clinical impact. The second step determines the clinical impact of an observed image quality, that is to repeat the scan or not. This clinical impact is based on the interpretation of the reporting radiologist as well as of the other multidisciplinary team members.

The second comment refers to the technical recommendations of PI-RADS. The PI-QUAL v. 1 assessment system includes technical parameters from the PI-RADS guidelines that have set minimal and optimal technical standards for MRI acquisition. It is important to note that compliance with these recommendations does not guarantee optimal quality imaging [5]. For example, a (slight) deviation of these technical recommendations in a particular situation can provide better quality images [12]. Therefore, one should identify sub-optimal image quality without scoring and without even partially linking it to the technical PI-RADS parameters. In order to be accepted by the radiological community, PI-QUAL needs to be-like PI-RADS-as simple as possible.

The PI-QUAL v.1 assessment system was developed in a single institution, and two radiologists from this center, a senior who trained the junior, have assessed the examination. This can affect the reproducibility and generalization of results to other readers and other centers. The scoring system should, therefore, be tested on different readers in different centers. These readers should be ideally trained, for example, through an online or paper-based lexicon, like the CORADS Score Practice initiatives for COVID-19 CT severity on the Grand Challenge platform (grand-chall enge.org) [13].

The proposed PI-QUAL v.1 assessment system is an essential starting tool to standardize the reporting of prostate image quality as objectively as possible. With further refinements, as mentioned above, and reproducibility and generalizability studies to confirm its high inter- and intra-reader agreement, the PI-QUAL assessment system can serve as an international standard. An international working group with representatives of ESUR and ESUI, among others, is currently working on an updated version of PI-QUAL (v.2), and testing it. Like the PI-RADS guidelines, the PI-QUAL guidelines will be a "living document" that will evolve with increasing clinical experience and scientific data.

Successful delivery of the MRI-guided PCa pathway requires imaging to be performed and reported to a sufficiently high level. Sub-optimal quality will have a negative impact on each component of the downstream MRI pathway. This first described PI-QUAL assessment system is of utmost importance. It will develop step by step into a reliable quality assessment tool, ensuring that the first step of the MRI pathway is as accurate as possible.

Funding The authors state that this work has not received any funding.

\section{Declarations}

Guarantor The scientific guarantor of this publication is J.O. Barentsz.

Conflict of interest The authors of this manuscript declare no relationships with any companies, whose products or services may be related to the subject matter of the article.

Statistics and biometry No complex statistical methods were necessary for this paper.

\section{Informed consent N/A.}

Ethical approval Institutional Review Board approval was not required because it is an editorial comment.

Methodology • editorial comment

\section{References}

1. Mottet N, van den Bergh RCN, Briers E et al (2021) EAU-EANMESTRO-ESUR-SIOG guidelines on prostate cancer-2020 update. Part 1: screening, diagnosis, and local treatment with curative intent. Eur Urol 79(2):243-262

2. van der Leest M, Cornel E, Israël B et al (2019) Head-to-head comparison of transrectal ultrasound-guided prostate biopsy versus multiparametric prostate resonance imaging with subsequent magnetic resonance-guided biopsy in biopsy-naive men with elevated prostate-specific antigen: a large prospective multicenter clinical study. Eur Urol 75(4):570-578

3. Ahmed HU, El-Shater Bosaily A, Brown LC et al (2017) Diagnostic accuracy of multi-parametric MRI and TRUS biopsy in prostate cancer (PROMIS): a paired validating confirmatory study. Lancet 389(10071):815-822

4. Rouvière O, Puech P, Renard-Penna R et al (2019) Use of prostate systematic and targeted biopsy on the basis of multiparametric MRI in biopsy-naive patients (MRI-FIRST): a prospective, multicentre, paired diagnostic study. Lancet Oncol 20(1):100-109 
5. Burn PR, Freeman SJ, Andreou A, Burns-Cox N, Persad R, Barrett T (2019) A multicentre assessment of prostate MRI quality and compliance with UK and international standards. Clin Radiol 74(11):894. e19-894.e25. https://doi.org/10.1016/j.crad.2019.03.026

6. Padhani AR, Schoots IG, Turkbey B, Giannarini G, Barentsz JO (2021) A multifaceted approach to quality in the MRIdirected biopsy pathway for prostate cancer diagnosis. Eur Radiol 31(6):4386-4389

7. de Rooij M, Israël B, Tummers M et al (2020) ESUR/ESUI consensus statements on multi-parametric MRI for the detection of clinically significant prostate cancer: quality requirements for image acquisition, interpretation and radiologists' training. Eur Radiol 30(10):5404-5416

8. Giganti F, Allen C, Emberton M, Moore CM, Kasivisvanathan V; PRECISION study group (2020) Prostate imaging quality (PIQUAL): a new quality control scoring system for multiparametric magnetic resonance imaging of the prostate from the PRECISION trial. Eur Urol Oncol 3(5):615-619

9. Giganti F, Kirkham A, Kasivisvanathan V et al (2021) Understanding PI-QUAL for prostate MRI quality: a practical primer for radiologists. Insights Imaging 12(1):59

10. Turkbey B, Rosenkrantz AB, Haider MA et al (2019) Prostate Imaging Reporting and Data System Version 2.1: 2019 Update of Prostate Imaging Reporting and Data System Version 2. Eur Urol 76(3):340-351
11. Giganti F, Dinneen E, Kasivisvanathan V et al (2021) Inter-reader agreement of the PI-QUAL score for prostate MRI quality in the NeuroSAFE PROOF trial. Eur Radiol. https://doi.org/10.1007/ s00330-021-08169-1

12. Leest MV, Israël B, Engels RRM, Barentsz JO (2020) Reply to Arnaldo Stanzione, Massimo Imbriaco, and Renato Cuocolo's Letter to the Editor re: Marloes van der Leest, Bas Israel, Eric Bastiaan Cornel, et al. High diagnostic performance of short magnetic resonance imaging protocols for prostate cancer detection in biopsynaive men: the next step in magnetic resonance imaging accessibility. Eur Urol 2019;76:574-81. Are we meeting our standards? Stringent prostate imaging reporting and data system acquisition requirements might be limiting prostate accessibility. Eur Urol 77(3):e58-e59

13. Prokop M, van Everdingen W, van Rees Vellinga T et al (2020) CO-RADS: a categorical CT assessment scheme for patients suspected of having COVID-19-definition and evaluation. Radiology 296(2):E97-E104

Publisher's note Springer Nature remains neutral with regard to jurisdictional claims in published maps and institutional affiliations. 